\title{
Influencia del tamaño de partícula de polvo sobre las propiedades mecánicas de la aleación PM Al7075 reforzada con SiCp
}

\author{
M. Torralba ${ }^{(*)}$, P. Adeva ${ }^{(*)}$, M. Lieblich $^{(*)}$, J. Ibáñez $^{(*)}$, G. Caruana $^{(*)}$, \\ A. García-Escorial ${ }^{(*)}$ y G. González-Doncel ${ }^{(*)}$
}

\begin{abstract}
Resumen Se ha estudiado la influencia del tamaño de partícula de polvo de la aleación de aluminio 7075, obtenido en condiciones de solidificación rápida (SR) por atomización con argón a alta presión, sobre las propiedades mecánicas a temperatura ambiente de un material compuesto 7075-15 \% vol. SiCp. Para preparar el material compuesto, se utilizaron tres tamaños distintos de partículas de polvo de la aleación: $<50 \mu \mathrm{m}, 50-100 \mu \mathrm{m}$ y $100-200 \mu \mathrm{m}$. Estas fracciones se mezclaron en un molino de bolas con un $15 \%$ vol. de partículas de $\mathrm{SiC},<5 \mu \mathrm{m}$, y luego se extruyeron en caliente. Las mejores propiedades del compuesto se obtienen cuando se utiliza el tamaño más pequeño. En este caso, se produce una clara mejoría de las propiedades mecánicas respecto del mismo material sin reforzar y obtenido por el mismo procedimiento, excepto para la ductilidad. Esta mejora se atribuye principalmente a una dispersión más homogénea de las partículas reforzantes de SiC.
\end{abstract}

Palabras clave: Materiales compuestos. Al7075-SiCp. Tamaño de partícula. Solidificación rápida. Molino de bolas.

\section{Influence of powder particle size on the mechanical properties of $\mathrm{SiCp}$ reinforced A.A.7075}

\begin{abstract}
The influence of powder particle size of 7075 aluminum on the room temperature mechanical properties of 7075-15 vol. \% SiCp has been studied. The aluminum powder was obtained by argon atomization under rapid solidification conditions. Composite materials were prepared from three different particle sizes, namely; $<50 \mu \mathrm{m}, 50-100 \mu \mathrm{m}$ and 100-200 $\mu \mathrm{m}$. These powders were blended with 15 vol \% of $\mathrm{SiC}$ particles $<5 \mu \mathrm{m}$ in size by ball milling and then, hot extruded. The best properties of the resulting composites correspond to the material prepared from the smallest particle size. For this case, a clear improvement of the mechanical properties, except for ductility, is obtained. This is attributed to a more homogeneous distribution of the reinforcing $\mathrm{SiC}$ particles.
\end{abstract}

Keywords: Composites. A.A.7075-SiCp. Particle size. Rapid solidification. Ball mill.

\section{INTRODUCCIÓN}

En las dos últimas décadas, se han investigado ampliamente los materiales compuestos (MC) de base aluminio con refuerzo discontinuo de carburo de silicio a causa de sus mejores propiedades mecánicas respecto de las aleaciones de aluminio. Propiedades mecánicas, tales como el módulo de Young o la resistencia a la tracción, mejoran considerablemente en estos materiales compuestos, sin

(*) Centro Nacional de Investigaciones Metalúrgicas, CENIM (CSIC), Avda. de Gregorio del Amo, 8. 28040-Madrid (España). aumentar de forma sensible la densidad, aunque a expensas de disminuir la ductilidad y la tenacidad. Estas mejoras, unidas a su facilidad de procesado y menor coste, respecto a los MC metálicos reforzados con fibra larga, hacen que estos materiales sean muy prometedores en las industrias del automóvil y aeroespacial (1-3).

Sin embargo, en las aleaciones de la serie 7XXX no se ha conseguido mejorar sus propiedades mecánicas añadiéndoles reforzantes de $\mathrm{SiC}$ (4-12). Por el contrario, se produce un cierto deterioro de estas propiedades. Así, en el caso de la aleación pulvimetalúrgica MB78 de Alcoa (de composición nominal: 
Al-7Zn-2Cu-2Mg-0,14Zr, muy similar a la 7091) en estado T6, cuando se la refuerza con un $20 \%$ vol. de partículas de $\mathrm{SiC}$ de $16 \mu \mathrm{m}$ y de $5 \mu \mathrm{m}$, el límite elástico baja (7). Igualmente, en la aleación 7075 reforzada con whiskers (20\% vol.), partículas (20\% vol.) o nódulos (30\% vol.) se produce un considerable descenso de su límite elástico (10).

Por tanto, resulta muy interesante encontrar una vía para mejorar las propiedades mecánicas de los materiales compuestos derivados de la serie 7XXX, dada la gran variedad de aplicaciones industriales de esta familia de aleaciones de aluminio. En este trabajo se muestra que estas mejoras se pueden conseguir mediante un refuerzo discontinuo de partículas de $\mathrm{SiC}$, siempre que se controlen cuidadosamente las variables de procesado. Se ha recurrido a técnicas pulvimetalúrgicas (PM), que permiten un buen control de la mezcla y de la microestructura final.

\section{MATERIALES Y PROCEDIMIENTO EXPERIMENTAL}

Se estudió la aleación 7075 (Al-5,6Zn-2,5Mg$1,6 \mathrm{Cu}-0,23 \mathrm{Cr}$ ) reforzada con $15 \%$ vol. de partículas de $\mathrm{SiC}$ de tamaño $<5 \mu \mathrm{m}$. La aleación 7075 se atomizó en el Atomizador de Gas de Alta Presión de Tobera Confinada (Leybold VIGA 2S) disponible en el CENIM. Como gas, se utilizó argón a una presión de 22-25 bar. Los polvos resultantes de la atomización presentaban microestructuras típicas de solidificación rápida. Estos polvos se tamizaron y se separaron por tamaños en tres fracciones de diámetro $d: d<50 \mu \mathrm{m}, 50 \mu \mathrm{m}<d<100 \mu \mathrm{m}$, y 100 $\mu \mathrm{m}<d<200 \mu \mathrm{m}$. Cada una de estas fracciones se mezcló con un $15 \%$ vol. de partículas de $\mathrm{SiC}$ de tamaño nominal $<5 \mu \mathrm{m}$ (Goodfellow). La mezcla se realizó, tras diversos ensayos, en un molino de bolas de alta energía, con bolas de $20 \mathrm{~mm}$ de diámetro y una relación 10:17 de material a bolas, a 150 r.p.m. durante $2 \mathrm{~h}$, con inversión de giro.

La consolidación de las mezclas se realizó, previa desgasificación, mediante extrusión en caliente a $480{ }^{\circ} \mathrm{C}$, con velocidad del empujador de $0,5 \mathrm{~mm} / \mathrm{s}$ y relación de extrusión de 24:1. Para comparar el efecto de las partículas de $\mathrm{SiC}$, también se extruyeron polvos de la aleación 7075 sin reforzar, con $d<$ $50 \mu \mathrm{m}$. Previamente, estos polvos habían sido tratados en el molino de bolas de la misma manera que las mezclas.

Las microestructuras de las barras extruidas de 7075-SiC se estudiaron mediante microscopía electrónica de barrido en secciones transversales y longitudinales. De las barras, se mecanizaron probetas de tracción de $30 \mathrm{~mm}$ de longitud útil y $6 \mathrm{~mm}$ de diámetro, a las que se dio el tratamiento térmico convencional T6 de $465^{\circ} \mathrm{C} / 90 \mathrm{~min}+$ temple +120 ${ }^{\circ} \mathrm{C} / 24 \mathrm{~h}$, para conseguir la resistencia máxima (11).
Los ensayos de tracción se realizaron a temperatura ambiente, con extensómetro y a velocidad inicial de $4 \times 10^{-4} \mathrm{~s}^{-1}$. Para la determinación del módulo dinámico de Young se utilizó un elasticímetro y probetas de $120 \mathrm{~mm}$ de longitud y 5,6 $\mathrm{mm}$ de diámetro.

\section{RESULTADOS Y DISCUSIÓN}

En la figura 1 se muestra la morfología de los tres tamaños de partículas de 7075 SR, que muestran una rugosidad superficial que indica una microestructura microcelular-dendrítica, que se afina al reducirse el tamaño partícula.

La distribución de las partículas de $\mathrm{SiC}$, después del proceso de mezcla, se muestra en la figura 2. Las zonas oscuras son aglomerados de partículas de $\mathrm{SiC}$ y las más claras aluminio. La figura $2 \mathrm{a}$ corresponde a la mezcla con polvo de diámetro $<50 \mu \mathrm{m}$, mientras que las figuras $2 b$ y $2 c$ corresponden a diámetros de 50-100 $\mu \mathrm{m}$ y $100-200 \mu \mathrm{m}$, respectivamente. Se observa que el proceso en el molino de bolas da lugar a una mezcla homogénea $\mathrm{Al} / \mathrm{SiCp}$, especialmente en el caso del menor tamaño de polvo. Las partículas de SiC se incrustan en la superficie de las partículas de polvo de aleación, que son mayores y más blandas. Este efecto se hace más evidente en el tamaño menor.

En la figura 3 se muestran secciones longitudinales de las barras obtenidas a partir de los tres tamaños de polvo. Puede apreciarse que la homogeneidad de la distribución de las partículas de $\mathrm{SiC}$ mejora a medida que el tamaño de polvo de la matriz de 7075 disminuye.

En la tabla I se presentan los valores del límite elástico, la resistencia a la tracción, el alargamiento hasta la rotura y el módulo de Young de nuestros materiales y, como comparación, los valores obtenidos por otros autores. Todas las propiedades mejoran al disminuir el tamaño de partícula de polvo, incluida la reproducibilidad de los ensayos. El valor del módulo es siempre mayor en los materiales reforzados que en el material no reforzado, y disminuye al aumentar el tamaño de polvo.

En general, los valores de las propiedades mecánicas encontradas en el presente trabajo son más altos que los mencionados en la literatura $(4-9,11$ y 12). El empeoramiento en las propiedades de los $\mathrm{MC}$, frente a las de los materiales sin reforzar, se atribuye a diferentes características microestructurales. Por ejemplo, Manoharan y Lewansdowski (6) creen que es debido a fractura de los reforzantes cerámicos. Indican, de acuerdo con Humphreys (5), que cuando un material compuesto con matriz de alta resistencia es deformado, la tensión en el reforzante cerámico aumenta considerablemente, dando lugar a un descenso en la capacidad de soportar cargas del material compuesto. 

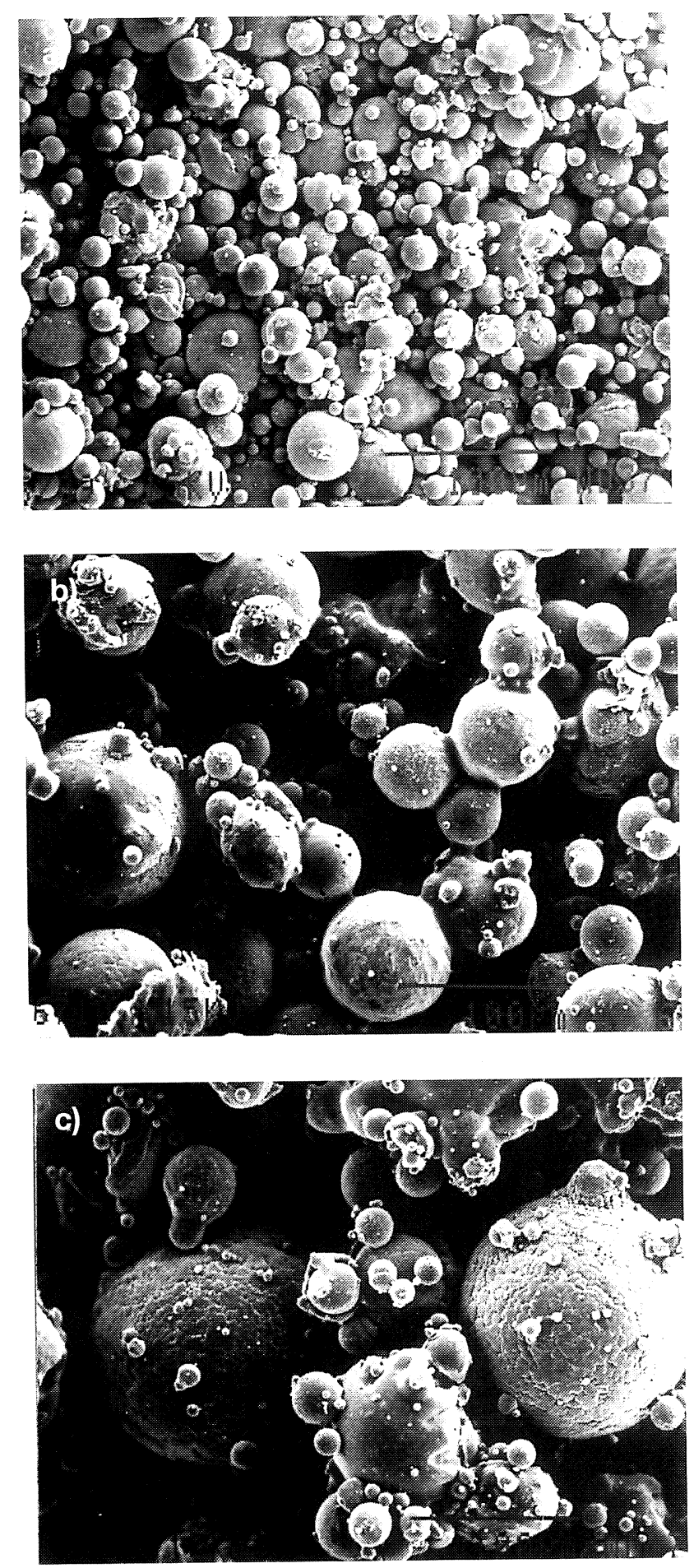

FIG. 1.- Morfología de los tres tamaños de polvo: a) $d<50 \mu \mathrm{m}$, b) $50 \mu \mathrm{m}<d<100 \mu \mathrm{m}$ y $c$ ) $100 \mu \mathrm{m}$ $<d<200 \mu \mathrm{m}$.

FIG. 1.- Morphology of the three powder particle sizes: a) ; $\mathrm{d}<50 \mu \mathrm{m}$, b) $50 \mu \mathrm{m}<\mathrm{d}<100 \mu \mathrm{m}$ and c) $100 \mu \mathrm{m}<\mathrm{d}<200 \mu \mathrm{m}$.

La nucleación de microgrietas en el reforzante depende acusadamente del tipo de matriz y del tamaño de las partículas de reforzante (12 y 13) pero, sobre todo, del tratamiento térmico y de la
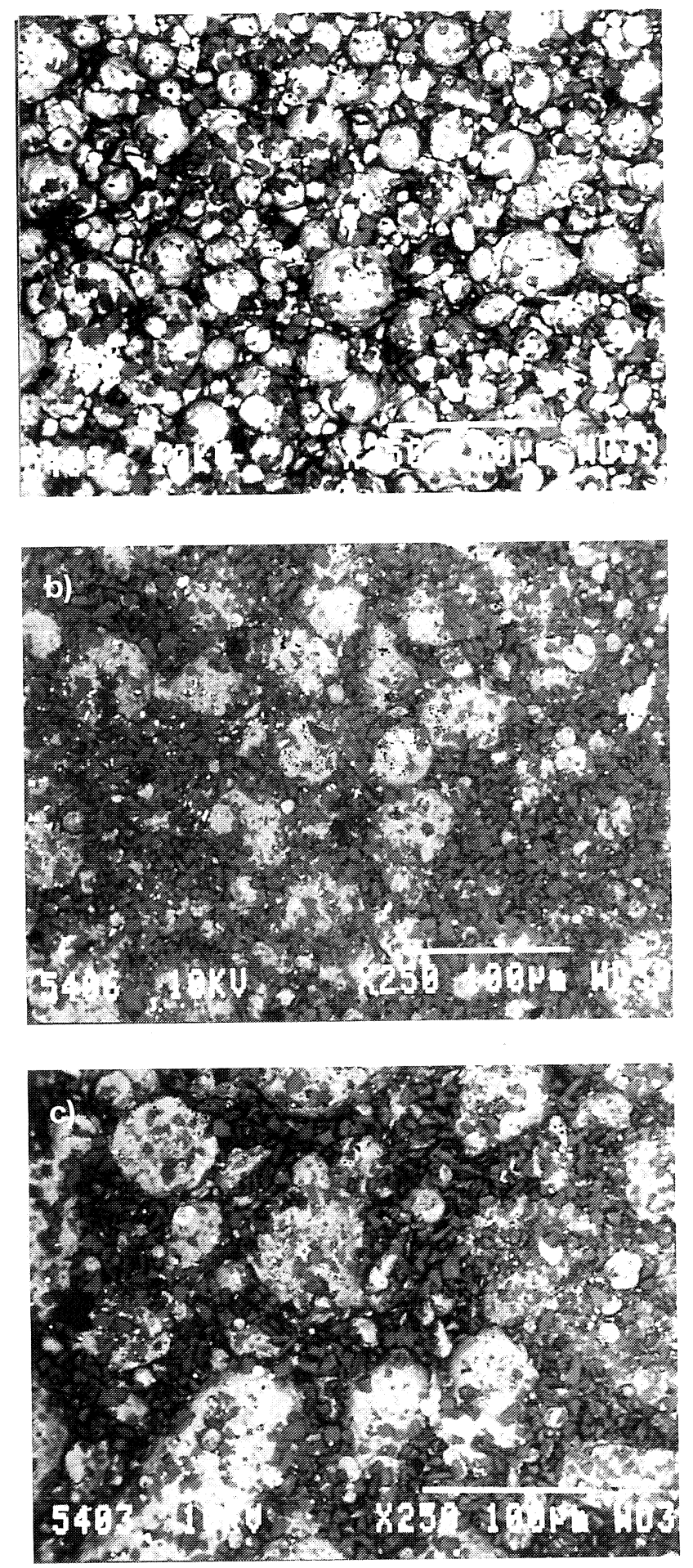

FIG. 2.- Homogeneización del material 7075/SiCp para los tres tamaños de polvo después del tratamiento en molino de bolas: a) $d<50 \mu \mathrm{m}$, b) 50 $\mu \mathrm{m}<d<100 \mu \mathrm{m}$ y c) $100 \mu \mathrm{m}<d<200 \mu \mathrm{m}$.

FIG. 2.- 7075-SiCp powder mixtures for the three powder particle sizes after homogenization by ball milling: a) $\mathrm{d}<50 \mu \mathrm{m}$, b) $50 \mu \mathrm{m}<d<100 \mu \mathrm{m}$ and c) $100 \mu \mathrm{m}<d<200 \mu \mathrm{m}$.

distribución de las partículas. Es muy posible que las fracturas se produzcan en regiones donde hay 

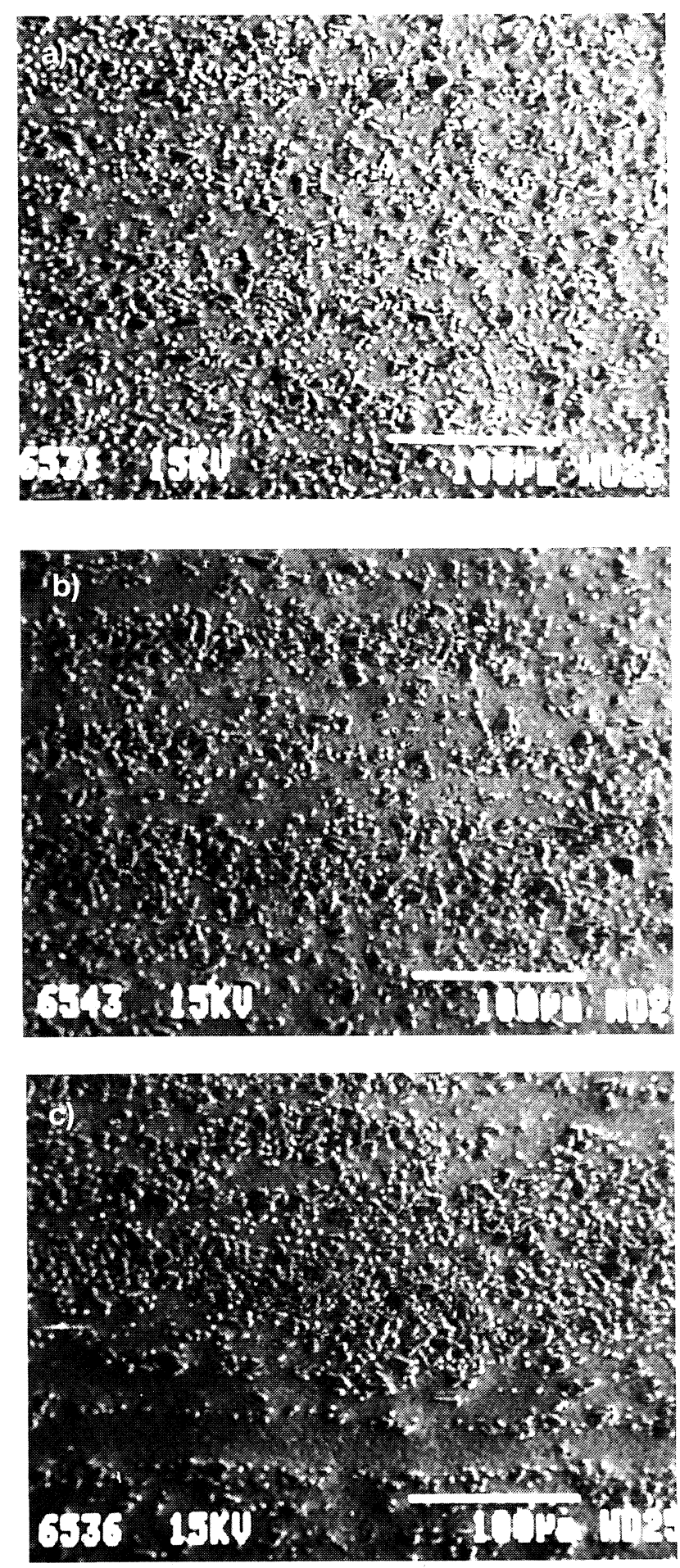

FIG. 3.- Secciones longitudinales de las barras extruidas a partir de las mezclas de $7075 / \mathrm{SiC}$ con distintos tamaños de polvo: a) $d<50 \mu \mathrm{m}$, b) $50 \mu \mathrm{m}$ $<d<100 \mu \mathrm{m} \mathrm{y} \mathrm{c)} 100 \mu \mathrm{m}<d<200 \mu \mathrm{m}$.

FIG. 3.- Longitudinal sections of the extruded bars from the 7075/SiC blends with the three particle sizes: a); $\mathrm{d}<50 \mu \mathrm{m}, b) 50 \mu \mathrm{m}<\mathrm{d}<100 \mu \mathrm{m}$ and c) $100 \mu \mathrm{m}<\mathrm{d}<200 \mu \mathrm{m}$.

acumulación de reforzantes. La figura 3 muestra que cuando se utilizan partículas de polvo $\operatorname{con} d<$
$50 \mu \mathrm{m}$, el SiC está repartido de forma muy homogénea. Esta ausencia de aglomerados de $\mathrm{SiCp}$ puede explicar que las partículas no se fracturen (7), lo que se traduce en una mejora, respecto del material sin reforzar, de $60 \mathrm{MPa}$ en el límite elástico, de $15 \mathrm{MPa}$ en la carga de rotura y de $35 \mathrm{GPa}$ en el módulo de Young. Por otra parte, parece que las partículas pequeñas de $\mathrm{SiC}(5 \mu \mathrm{m})$ son menos propensas a fracturarse durante el ensayo, ya que tienen menos probabilidades de presentar defectos donde se inicie la fractura (14).

La mayoría de los autores (4-12) encuentran que los materiales reforzados con partículas o whiskers de $\mathrm{SiC}$ con matriz de aleación de Al7075 ó MB78 tienen peores propiedades que los materiales sin reforzar, tanto en lo referente al límite elástico como a la carga de rotura y, especialmente, en relación al alargamiento hasta la rotura (Tabla I).

En cuanto a la aleación 7075 reforzada con partículas de $\mathrm{SiC}$, los valores de las propiedades obtenidos en el presente trabajo, para $d<50 \mu \mathrm{m}$, son claramente superiores a los encontrados en la bibliografía. Además, es el único material que posee un límite elástico superior al de la aleación 7075 sin reforzar (15). El valor del alargamiento es comparable al de los otros materiales mencionados en la bibliografía. Esta mejora en las propiedades mecánicas puede atribuirse a una mezcla muy homogénea de las partículas reforzantes de $\mathrm{SiC}$ y a que éstas han sido mezcladas de tal manera que no se agrietan.

\section{CONCLUSIONES}

Del estudio del comportamiento mecánico de los compuestos PM Al7075-15 \% vol. SiCp, obtenidos a partir de tres tamaños de partículas de polvo de aleación, se ha llegado a las siguientes conclusiones:

Las propiedades mecánicas de los materiales compuestos estudiados empeoran a medida que el tamaño de partícula de polvo de la aleación aumenta. El material compuesto obtenido a partir del tamaño de polvo menor, $d<50 \mu \mathrm{m}$, es el que da lugar a las mejores propiedades.

La reproducibilidad de los resultados de los ensayos de tracción también mejora al disminuir el tamaño de las partículas de polvo. Para el material con $d<50 \mu \mathrm{m}$, la reproducibilidad es semejante a la del material PM sin reforzar.

Excepto el alargamiento en tracción, las propiedades mecánicas estudiadas del material con $d<50$ $\mu \mathrm{m}$ mejoran con respecto a la misma aleación sin reforzar, tratada en las mismas condiciones, y a las obtenidas por otros autores.

La mejora de las propiedades mecánicas observada en estos materiales se atribuye a la distribución homogénea de las partículas de $\mathrm{SiC}$ en la 
TABLA I.- Propiedades de tracción de materiales compuestos con matriz de aleaciones de aluminio de la serie 7XXX reforzadas con $\mathrm{SiC}$

TABLE I.- Tensile properties of SiCp reinforced 7XXX group aluminium alloys

\begin{tabular}{|c|c|c|c|c|c|}
\hline Autores y Ref. & $\begin{array}{l}\text { Material } \\
+ \\
\mathrm{SiC} \%-\mathrm{SiC} \mu \mathrm{m}\end{array}$ & $\begin{array}{l}\text { L. elást. } \\
\mathrm{MPa}\end{array}$ & $\begin{array}{l}\text { R. trac. } \\
\mathrm{MPa}\end{array}$ & $\begin{array}{l}\text { Alarg. } \\
(\%)\end{array}$ & $\begin{array}{l}\text { Mód. } \\
\text { Young } \\
\text { (GPa) }\end{array}$ \\
\hline $\begin{array}{l}\text { Lewandowski (7) } \\
\text { Manoharan (12) }\end{array}$ & $\begin{array}{l}\text { MB78 } \\
\text { sin refuerzo }\end{array}$ & $\begin{array}{l}554 \\
553\end{array}$ & $\begin{array}{l}589 \\
594\end{array}$ & $\begin{array}{l}17 \\
19\end{array}$ & \\
\hline Lewandowski (7) & $\begin{array}{l}\text { MB78-20-5 } \\
\text { MB78-20-15 }\end{array}$ & $\begin{array}{l}543 \\
518\end{array}$ & $\begin{array}{l}598 \\
583\end{array}$ & $\begin{array}{l}4 \\
5\end{array}$ & \\
\hline Manoharan (12) & $\begin{array}{l}\text { MB78-20-15 } \\
\text { MB78-20-5 }\end{array}$ & $\begin{array}{l}418 \\
406\end{array}$ & $\begin{array}{l}473 \\
459\end{array}$ & $\begin{array}{l}3 \\
3\end{array}$ & \\
\hline Manoharan (6) & MB78-15-5 & 417 & 569 & 4,9 & \\
\hline Hong y Gray (11) & $\begin{array}{l}\text { MB78-20-5 } \\
\text { MB78-20-15 }\end{array}$ & $\begin{array}{l}405 \\
392\end{array}$ & & & \\
\hline Shang y Ritchie (8) & $\begin{array}{l}\text { MB78-15-15 } \\
\text { MB78-20-5 }\end{array}$ & $\begin{array}{l}490 \\
500 \\
\end{array}$ & $\begin{array}{l}535 \\
560\end{array}$ & $\begin{array}{l}1,1 \\
1,8 \\
\end{array}$ & $\begin{array}{l}99 \\
101\end{array}$ \\
\hline $\begin{array}{l}\text { Alumin. Alloys (15) } \\
\text { Este trabajo }\end{array}$ & $\begin{array}{l}7075-\mathrm{T} 6 \\
\text { sin refuerzo }\end{array}$ & $\begin{array}{l}503 \\
503 \pm 19\end{array}$ & $\begin{array}{l}572 \\
657 \pm 20\end{array}$ & $\begin{array}{l}11 \\
12,6\end{array}$ & $\begin{array}{l}71 \\
74 \pm 4\end{array}$ \\
\hline Este trabajo & $d<50 \mu \mathrm{m}$ & $565 \pm 7$ & $671 \pm 13$ & $2,8 \pm 0,5$ & $108 \pm 6$ \\
\hline Este trabajo & $50-100 \mu \mathrm{m}$ & $536 \pm 16$ & $580 \pm 20$ & $1,0 \pm 0,4$ & $101 \pm 14$ \\
\hline Este trabajo & $100-200 \mu \mathrm{m}$ & $535 \pm 12$ & $575 \pm 25$ & $0,9 \pm 0,8$ & $93 \pm 7$ \\
\hline Sritharan (4) & $7075-10-$ & 420 & 516 & 3 & \\
\hline Humphreys (5) & 7075-20- & 450 & 550 & $3-4$ & \\
\hline McDanels (10) & $7075-30-5$ & 390 & 420 & 5 & \\
\hline
\end{tabular}

Nota. Todos los materiales están en estado T6. El primer número que sigue a la designación de la aleación indica el \% vol. de SiC reforzante, y el segundo número se refiere al tamaño de las partículas de reforzante en $\mu \mathrm{m}$.

matriz y a que las partículas de $\mathrm{SiC}$ pequeñas $(<5$ $\mu \mathrm{m})$ tienen menos propensión a fracturarse durante el ensayo que las partículas más grandes.

\section{Agradecimiento}

Este trabajo ha sido realizado bajo contrato con la empresa Tecnología y Gestión de la Innovación, S.A. (TGI), a la que se agradece la autorización para publicarlo.

\section{REFERENCIAS}

(1) Koczak, M.J., Khatri, S.C., Allison, J.E. y Bader, M.G. Fundamentals of Metall Matrix Composites (Eds. Suresh, S., Mortensen, A. y Needleman, A. ButterworthHeinemann, MA. 1993: 297-326.

(2) Allison, J.E. y Cole, G. JOM, 45 (1), $1993: 19-25$.

(3) McKimpson, M.G., Pohlenz, E.L. y Thompson, S.R. JOM, 45 (1), 1993: 26-29.

(4) Sritharan, T., Xia, K., Heathcock, J. y Mihelich, J. Metal \& Ceramic Matrix Composites: Processing, Modeling \& Mechanical Behavior, (Eds. Bhagat, R.B.,
Clauer, A.H., Kumar, P. y Ritter, A.M.), TMS, Warrendale, PA, (EE.UU.) $1990: 13-22$.

(5) Humphreys, F. J. Mechanical and Physical Behavior of Metallic and Ceramic Composites, (Eds. Anderson, S. I. et al.). Riso Natn. Lab. Dinamarca. 1988 : 51-74.

(6) Manoharan, M. y Lewandowski, J.J. Acta Metall. Mater., 38 (3), 1990: 489-496.

(7) Lewandowski, J.J., Liu, C. y Hunt, W.H. Jr., Processing and Properties for Powder Metallurgy Composites, (Eds. Kumar, P., Vedula, K. y Ritter, A.), TMS-AIME, Warrendale, PA. (EE.UU.) 1988: 117-138.

(8) Shang, J.K. y Ritchie, R.O. Acta Metall., 37, 1989: 2.267-2.278

(9) Dionne, S., Krishnadev, M.R. y Bouchard, R. Metal \& Ceramic Matrix Composites: Processing, Modeling \& Mechanical Behavior, (Eds. Bhagat, R.B., Clauer, A.H., Kumar, P. y Ritter, A.M.), TMS, Warrendale, PA. (EE.UU.) 1990: 243-251.

(10) McDanels, D.L. Metall. Trans. A, 16A, 1985: 1.105-1.115.

(11) Hong, S.I. y Gray III, G.T. Acta. Metall. Mater., 40 (12), 1992: 3.299-3.315.

(12) Manoharan, M. y Lewandowski, J.J. Mater. Sci. Eng. A, A150, 1992: 179-186.

(13) Singh, P.M. y LeWAndowski, J.J. Metall. Trans. A, 24A 1993: 2.531-2.543.

(14) Wang, B., Janowski, G.M. y Patterson, B.R. Metall. Mater. Trans. A, 26A, 1995: 2.457-2.467.

(15) Aluminium and Aluminium Alloys. ASM Specialty Handbook (Ed. Davis, J.R.). ASM International. 1994: 698. 